\title{
Enhancing Brain Lesions after Endovascular Treatment of Aneurysms
}

\author{
J.P. Cruz, T. Marotta, C. O’Kelly, M. Holtmannspötter, G. Saliou, R. Willinsky, T. Krings, and R. Agid
}

\begin{abstract}
SUMMARY: Complications of endovascular therapy of aneurysms mainly include aneurysm rupture and thromboembolic events. The widespread use of MR imaging for follow-up of these patients revealed various nonvascular complications such as aseptic meningitis, hydrocephalus, and perianeurysmal brain edema. We present 7 patients from 5 different institutions that developed MR imagingenhancing brain lesions after endovascular therapy of aneurysms, detected after a median time of 63 days. The number of lesions ranged from 4-46 (median of 10.5), sized 2-20 mm, and were mostly in the same vascular territory used for access. Three patients presented with symptoms attributable to these lesions. After a median follow-up of 21.5 months, the number of lesions increased in 2, was stable in 1, decreased in 3, and disappeared in 1 . The imaging and clinical characteristics suggested a foreign body reaction. We could find no correlation to a specific device, but a possible source may be the generic hydrophilic coating.
\end{abstract}

ABBREVIATION: EVT = endovascular therapy

$\mathbf{E}$ ndovascular therapy (EVT) is commonly used as a first-line therapy for intracranial aneurysms. ${ }^{1,2}$ The complication rate of this procedure is relatively low ${ }^{3}$ and mostly includes thromboembolic events and intraprocedure aneurysm rupture. ${ }^{4}$ Less common nonvascular complications exist and include hydrocephalus and perianeurysmal edema. ${ }^{5-7}$ There are isolated reports of foreign body emboli to the brain parenchyma after diagnostic and therapeutic cerebral angiograms, but only a few include the imaging characteristics of these lesions. ${ }^{8-11}$ We describe a short series of nonischemic, enhancing parenchymal lesions appearing after EVT of intracranial aneurysms.

\section{Case Series}

We report cases from 5 different institutions. The authors at each of the 5 participating centers submitted the demographic data,

Received January 30, 2014; accepted after revision March 14.

From the Radiology Department (J.P.C.), Hospital Clínico de la Pontificia Universidad Católica de Chile, Santiago, Chile; Divisions of Neuroradiology (T.M.), St. Michael's Hospital, Toronto, Ontario, Canada; Department of Surgery (C.O.), University of Edmonton, Edmonton, Alberta, Canada; Division of Interventional Neuroradiology (M.H.), Københavns Universitet, Copenhagen, Denmark; Service de Neuroradiologié (G.S.), Hôpital de Bicêtre, Kremlin Bicêtre, France; and Toronto Western Hospital (R.W., T.K., R.A.), Toronto, Ontario, Canada.

Please address correspondence to Ronit Agid, MD, Department of Medical Imaging, Toronto Western Hospital, 399 Bathurst St., 3 McLaughlin wing, Room 425, Toronto, ON, M5T 2S8, Canada; e-mail: ronit.agid@uhn.ca

- Indicates open access to non-subscribers at www.ajnr.org

三 Indicates article with supplemental on-line table.

http://dx.doi.org/10.3174/ajnr.A3976 aneurysm characteristics, procedure type, devices used, and MR characteristics of the lesions as well as the imaging and clinical findings during follow-up. Analysis of the submitted data was performed by 2 of the authors (J.P.C. and R.A.).

A total of 7 cases of brain enhancing lesions after EVT were collected (6 women, 1 man) ranging from 32 to 71 years of age (median age 54). Six aneurysms were located in the anterior circulation and 1 in the posterior circulation. Three aneurysms were ruptured and 4 were unruptured.

The procedures included 2 conventional aneurysm coilings, 2 balloon-assisted coilings, 1 stent-assisted coiling, and 2 cases of flow-diverter stents. All patients had a single femoral access. A triaxial system was used in all but 2 patients, 1 of them with a posterior circulation aneurysm. Bare platinum coils were used in 3 of the 4 coiling procedures. Modified platinum coils were used in only 1 patient. None of the patients treated with flow diversion $(n=2)$ had adjuvant coiling. We could not identify 1 specific device that was predominantly used in all cases (On-line Table).

The median time from procedure to lesion detection on MR was 63 days (range $43-118$ days, interquartile range $=35.5$ days). Four patients (2 ruptured, 2 unruptured) had an early postprocedure MR (ie, within 7 days from the procedure); 3 showed few restricted DWI lesions in the vascular territory used for access (Fig 1) that disappeared in follow-up as expected for ischemic lesions after EVT. ${ }^{12}$ All the other patients had the first postprocedure MR performed later as part of the routine follow-up. All but 1 patient had a contrast-enhanced MR imaging. The number of enhancing lesions per patient ranged from 4 to 46 (median 10.5, interquartile range $=33.5$ ), the 

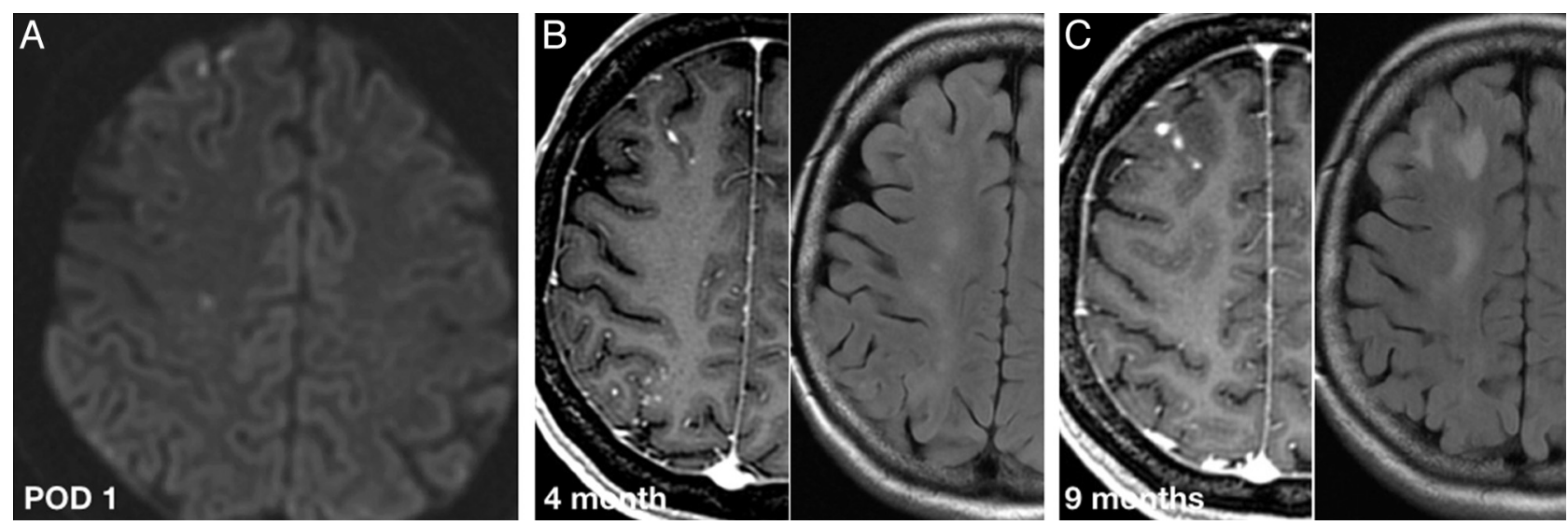

FIG 1. A 54-year-old woman with a right supraclinoid ICA unruptured aneurysm treated with a flow diverter. Axial DWI on postoperative day (POD) $1(A)$, contrast-enhanced T1-weighted and T2-FLAIR at 4 months $(B)$, and 9 months after procedure (C). POD 1 MR image shows a few asymptomatic DWI lesions in the frontal lobe. Four-month follow-up MR shows subcortical enhancing lesions in both the frontal and parietal lobes, far more numerous than the DWI lesions seen in POD 1. The enhancing lesions and the perilesional edema persist in the 9-month follow-up MR imaging, which is not the expected evolution for ischemic lesions. Foreign body reaction was the presumed diagnosis after all clinical and laboratory work-up was negative for infection.

size of the lesions ranged from 2-20 mm in diameter. Most lesions showed solid enhancement. Rim enhancement was seen only in lesions larger than $5 \mathrm{~mm}$. Lesions with rim enhancement were seen in 2 patients ( 2 lesions in each patient), and showed central restricted diffusion on DWI. Gradient recalled-echo signal dephasing was seen in 5 patients (total number of dephasing lesions $=14$; range $1-4$ ). Perilesional edema was seen in all patients, but varied in extent from lesion to lesion. All patients had subcortical lesions, but only 2 also showed deep gray matter lesions ( 1 anterior circulation and 1 posterior circulation aneurysm). The enhancing lesions were limited to the vascular territory of the artery used to access the aneurysm in 6 cases. In 1 case, the lesions were mostly in the expected vascular territory, with few isolated lesions elsewhere. The sample size did not allow for any subgroup analysis regarding procedure type and characteristics of the enhancing lesions.

Four patients had no neurologic symptoms related to the brain lesions (incidental finding on MR follow-up), 1 patient presented with paresthesias that were attributed to seizure-like activity, 1 patient had gait disturbances associated with posterior fossa lesions, and 1 had mild fluctuating central facial nerve palsy, mild arm weakness, and speech disturbances. Two of the asymptomatic patients had coexisting painless aseptic secretion and swelling at the arterial puncture site. None of the patients presented with hydrocephalus or with meningeal symptoms. In all patients, septic emboli were ruled out on clinical grounds and after an extensive work-up that included complete blood count, lumbar puncture, and echocardiography.

Treatment strategies for these lesions varied. Of the 4 neurologically asymptomatic patients, 2 received no treatment, 1 received empiric antibiotic therapy for 14 days, and 1 received both empiric antibiotics and steroids, with no changes in the imaging or clinical findings during follow-up. Of the 3 symptomatic patients, 1 received no treatment, 1 patient with presumed seizurelike activity received levetiracetam resulting in resolution of the symptoms, and 1 received combined antiobiotic therapy for 6 months, but lesions persisted in next follow-up (Fig 2). In the patient treated with levetiracetam, the drug was discontinued after 6 weeks and the patient remained asymptomatic, but the brain lesions remained unchanged. None of the patients underwent biopsy or surgical resection of the lesions.

Follow-up imaging including brain MR imaging and MRA was available for all 7 patients. Median follow-up was 21.5 months (range: 5-63 months, interquartile range $=11.25$ months). Within the follow-up period, the enhancing lesions disappeared in 1 case, decreased in number in 3 cases (Fig 2), were unchanged in 1 case, and increased in number in 2 cases. Perilesional edema fluctuated during the follow-up period, but on last follow-up was better in 5 cases, stable in 1 case, and showed a mixed response in 1 case. None of the cases showed continuing or new restricting lesions on DWI. The 4 rim-enhancing lesions evolved to a solid pattern of enhancement (Fig 3). Of the 14 lesions with gradient recalled-echo signal dephasing, 1 disappeared, 3 showed interval decrease in size, and the rest remained unchanged. All patients but 1 , including those initially symptomatic, had no neurologic symptoms attributable to the brain lesions at their last clinical appointment. None of the patients developed new or worsening symptoms.

\section{DISCUSSION}

It is reasonable to believe that the enhancing brain lesions we describe represent an embolic phenomenon as they were restricted in all but 1 case to the vascular territory of the catheterized arteries. In addition, lesions were predominately located in the cortico-subcortical region where small emboli are more likely to get lodged.

Clinically silent emboli are a common known occurrence of diagnostic cerebral angiograms and neuroendovascular procedures, occuring in approximately $13 \%-53 \%$ of cases. ${ }^{12,13}$ Inadvertent foreign body emboli is a far less known complication, first described in $1960 .{ }^{14}$ Subsequent reports mostly focused on this phenomenon as a cause of postprocedural ischemia. ${ }^{11}$ More recently, hydrophilic polymer emboli have been reported as a cause of brain infarction after EVT. ${ }^{10,15}$ In a more recent report, intraprocedural foreign body emboli of one of the water-soluble polymers used for hydrophilic coating (polyvinylpyrrolidone or PVP) have been associated with posttreatment delayed ipsilateral 

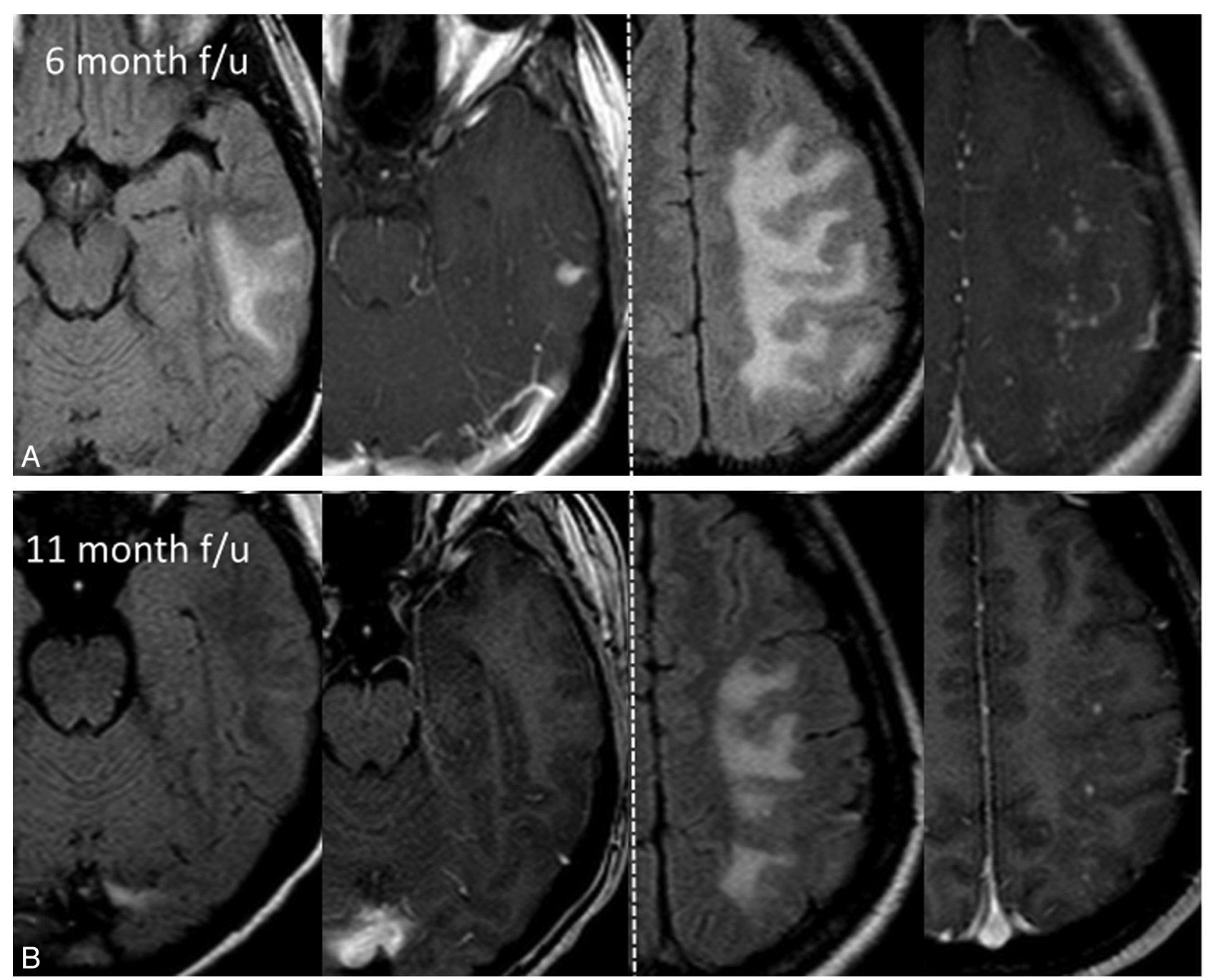

FIG 2. A 32-year-old woman with an unruptured left MCA aneurysm treated with balloon-assisted coiling. Axial FLAIR and axial T1-weighted contrast-enhanced MR images at 6 months $(A)$ and 11 months $(B)$ follow-up show multiple enhancing cortico-subcortical lesions in the distal MCA territory surrounded by vasogenic edema. Combined antibiotic therapy was initiated after $(A)$ with only partial improvement in the next follow-up. Note how the middle temporal gyrus lesion disappears over time while the middle frontal lesions only decrease in number.

intraparenchymal hemorrhage after flow diversion. ${ }^{16}$ The first reported brain parenchymal foreign body reaction presenting as MR imaging-enhancing lesions after EVT was published by Fealey et $\mathrm{al}^{8}$ in a 58-year-old woman with a ruptured right ICA aneurysm. This patient presented 9 months after uneventful coiling with seizures and 3 associated rim-enhancing brain lesions in the postcentral gyrus. After empiric antibitotic treatment with no change in the brain lesions, a brain biopsy was performed. The pathology specimen revealed granulomatous foreign body reaction and trapped filaments of hydrophilic coating polymer. Similar lesions were found in the arterial access site of the other 2 patients described in this case report.

It is likely that the number of diagnosed cases of brain foreign body granulomas after neuroendovascular procedures is increasing in recent years as MR and MRA widely replace the use of DSA for follow-up of aneurysms after EVT. Surprisingly, recent trials that have used MR imaging for follow-up do not comment on the presence or absence of enhancing lesions. ${ }^{2}$ Specifically, the 2 recent trials that used modified platinum coils where nonvascular complications were first described made no mention regarding subacute enhancing brain lesions in their published data. ${ }^{17,18}$
In our case series, the lesions were diagnosed on MR relatively late (after a median time of 63 days) and only a few lesions were restricted on DWI; thus, acute or subacute ischemia is not considered a plausible diagnosis. In patients for whom early postprocedure MR imaging was available, there were a few isolated DWI restricting lesions, but no early enhancing lesions were documented. All of our patients had perilesional edema, which prompted a more complete brain MR imaging protocol, and underwent extensive clinical and laboratory work-up to rule out an infectious etiology (ie, septic emboli). Moreover, the rare reports of brain abscesses after coiling, all presented with positive biochemical markers of CNS infection or isolated pathogen on CSF cultures. ${ }^{19-21}$ The 4 lesions in our series that showed diffusion restriction and rim enhancement could be in keeping with aseptic abscesses, which have been described in foreign body reaction after surgery. ${ }^{22-24}$ All these lesions evolved from a rim-enhancing pattern to a solid enhancement pattern in follow-up and the diffusion restriction disappeared (Fig 3). This appearance could represent evolution from an aseptic abscess to the more chronic form of a foreign body granuloma. 

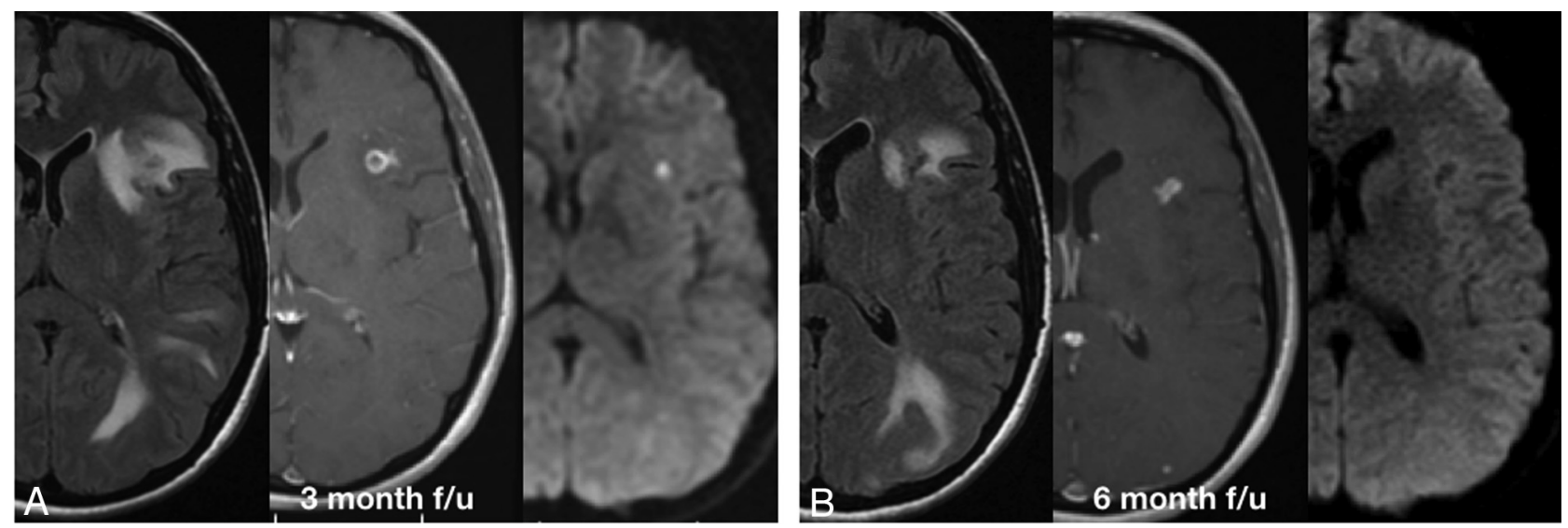

FIG 3. A 51-year-old woman with an asymptomatic intradural left cavernous ICA aneurysm treated with a flow diverter. Axial contrast-enhanced T1-weighted, T2-FLAIR, and DWI brain MR images at 3 months $(A)$ and 6 months $(B)$. A, Representation of 1 of the rim-enhancing lesions in the frontal operculum, with significant perilesional edema and restricted diffusion. An infectious cause was ruled out on clinical grounds and a foreign body reaction was considered as the most possible cause. The patient received 6 weeks of combined oral antibiotic therapy and remained asymptomatic. $B$, At 6-month follow-up, the lesions show a solid pattern of enhancement, with no restricted diffusion and only partial improvement of the edema. This was considered to represent the evolution of an aseptic abscess into a foreign body granuloma. The lesions and the surrounding edema decrease slowly in size but at 2-year follow up still exist (not shown).

Local foreign body reaction at the puncture site was described $(2.8 \%-5.0 \%)$ in the cardiology literature after radial access by using long hydrophilic coated sheaths. ${ }^{25-27}$ Two of our patients presented with aseptic secretion and local inflammation at the puncture site that did not respond to the initial antibiotic treatment, and eventually resolved spontaneously over a few months. Pathologic specimen was not obtained in these cases; however, the clinical picture corresponds to a foreign body skin reaction. Whether this makes the host more prone to developing a foreign body reaction to hydrophilic coating in the brain is unclear.

Our small case series is limited to EVT for aneurysms, which does not mean that this phenomenon cannot be seen in other neurovascular procedures. It is possible that all of our cases are aneurysmal due to the need to navigate distally toward the aneurysm and use coaxial catheters with friction between coaxial catheters and between the coils, stents, and the microcatheter lumen during these procedures. This might increase the risk of hydrophilic coating dislodgement. Aneurysm coiling is also the most common procedure in the institutions participating in this report and all participating institutions use MRA for follow-up, allowing depiction of asymptomatic lesions.

There are several limitations to this series. First, we report a limited series of incidental cases and not a systematic review of all cases performed, thus the exact incidence of this complication cannot be established. We can presume that it is a rare occurrence given that cases were submitted from 5 different referral neurovascular centers with an average of 95 aneurysms per center per year. One of the patients presented 5 years ago and the rest in the last 3 years with no other diagnosed cases in this time period. The timing of the initial and follow-up MR imaging varies widely from case to case and between institutions. Thus, a conclusion regarding the exact timing of lesion development is limited. In addition, none of our cases are proved by surgical biopsy, as lesion resection was not considered necessary, and the diagnosis was made on the basis of clinical and imaging features.

\section{CONCLUSIONS}

Enhancing brain lesions are a rare complication after aneurysm EVT. We did not find this complication exclusive to the use of any particular device. Imaging and clinical characteristics suggest a foreign body reaction such as aseptic abscess and granuloma. We suggest that the cause of these lesions might be shedding of hydrophilic coating from endovascular devices into the blood stream.

Disclosures: Thomas Marotta—UNRELATED: Consultancy: Proctor for Pipeline embolization device; Patents (planned, pending or issued): Eclips flow diverter. Cian O'Kelly-UNRELATED: Other: Proctor for Covidien for Pipeline insertion. Markus Holtmannspötter-UNRELATED: Consultancy: MicroVention, Covidien, Sequent Medical; Payment for Lectures (including service on speakers bureaus): MicroVention, Covidien, Sequent Medical.

\section{REFERENCES}

1. Molyneux AJ, Kerr RS, Birks J, et al. Risk of recurrent subarachnoid haemorrhage, death, or dependence and standardised mortality ratios after clipping or coiling of an intracranial aneurysm in the International Subarachnoid Aneurysm Trial (ISAT): long-term follow-up. Lancet Neurol 2009;8:427-33

2. Spetzler RF, McDougall CG, Albuquerque FC, et al. The Barrow ruptured aneurysm trial: 3-year results. J Neurosurg 2013;119:146-57

3. Pierot L, Wakhloo AK. Endovascular treatment of intracranial aneurysms: current status. Stroke 2013;44:2046-54

4. Pierot L, Cognard C, Spelle L, et al. Safety and efficacy of balloon remodeling technique during endovascular treatment of intracranial aneurysms: critical review of the literature. AJNR Am J Neuroradiol 2012;33:12-15

5. Fanning NF, Willinsky RA, ter Brugge KG. Wall enhancement, edema, and hydrocephalus after endovascular coil occlusion of intradural cerebral aneurysms. J Neurosurg 2008;108:1074-86

6. Horie N, Kitagawa N, Morikawa M, et al. Progressive perianeurysmal edema induced after endovascular coil embolization. Report of three cases and review of the literature. J Neurosurg 2007;106: 916-20

7. Marchan EM, Sekula RF, Jr, Ku A, et al. Hydrogel coil-related delayed hydrocephalus in patients with unruptured aneurysms. J Neurosurg 2008;109:186-90

8. Fealey ME, Edwards WD, Giannini C, et al. Complications of endo- 
vascular polymers associated with vascular introducer sheaths and metallic coils in 3 patients, with literature review. Am J Surg Pathol 2008;32:1310-16

9. Mehta RI, Mehta RI, Fishbein MC, et al. Intravascular polymer material after coil embolization of a giant cerebral aneurysm. Hum Pathol 2009;40:1803-07

10. Mehta RI, Mehta RI, Solis OE, et al. Hydrophilic polymer emboli: an under-recognized iatrogenic cause of ischemia and infarct. Mod Pathol 2010;23:921-30

11. Shannon P, Billbao JM, Marotta T, et al. Inadvertent foreign body embolization in diagnostic and therapeutic cerebral angiography. AJNR Am J Neuroradiol 2006;27:278-82

12. Bendszus M, Koltzenburg M, Burger R, et al. Silent embolism in diagnostic cerebral angiography and neurointerventional procedures: a prospective study. Lancet 1999;354:1594-97

13. Hill MD, Martin RH, Mikulis D, et al. Safety and efficacy of NA-1 in patients with iatrogenic stroke after endovascular aneurysm repair (ENACT): a phase 2, randomised, double-blind, placebo-controlled trial. Lancet Neurol 2012;11:942-50

14. Silberman J, Cravioto H, Feigin, I. Foreign body emboli following cerebral angiography. Trans Am Neurol Assoc 1960;85:123-25

15. Barnwell SL, D'Agostino AN, Shapiro SL, et al. Foreign bodies in small arteries after use of an infusion microcatheter. AJNR Am J Neuroradiol 1997;18:1886-89

16. Hu YC, Deshmukh VR, Albuquerque FC, et al. Histopathological assessment of fatal ipsilateral intraparenchymal hemorrhages after the treatment of supraclinoid aneurysms with the Pipeline embolization device. J Neurosurg 2014;120:365-74

17. Coley S, Sneade M, Clarke A, et al. Cerecyte coil trial: procedural safety and clinical outcomes in patients with ruptured and unruptured intracranial aneurysms. AJNR Am J Neuroradiol 2012;33: $474-80$

18. White PM, Lewis SC, Gholkar A, et al. Hydrogel-coated coils versus bare platinum coils for the endovascular treatment of intracranial aneurysms (HELPS): a randomised controlled trial. Lancet 2011; 377:1655-62

19. AL-Okaili, R, Patel SJ. Brain abscess after endovascular coiling of a saccular aneurysm: case report. AJNR Am J Neuroradiol 2002; 23:697-99

20. Jenkinson MD, Javadpour M, Nixon T, et al. Intracerebral abscess formation following embolisation of an internal carotid artery aneurysm using Guglielmi detachable coils. Acta Neurochir (Wien) 2003;145:703-05; discussion 705-06

21. Kirollos RW, Bosma JJ, Radhakrishnan J, et al. Endovascularly treated cerebral aneurysm using Guglielmi detachable coils acting as a nidus for brain abscess formation secondary to salmonella bacteremia: case report. Neurosurgery 2002;51:234-37; discussion 237-38

22. Jang SW, Kim SJ, Kim SM, et al. MR spectroscopy and perfusion MR imaging findings of intracranial foreign body granuloma: a case report. Korean J Radiol 2010;11:359-63

23. Kothbauer KF, Jallo GI, Siffert J, et al. Foreign body reaction to hemostatic materials mimicking recurrent brain tumor. Report of three cases. J Neurosurg 2001;95:503-06

24. Yoon MA, Kim E, Kwon BJ, et al. Muslinoma and muslin-induced foreign body inflammatory reactions after surgical clipping and wrapping for intracranial aneurysms: imaging findings and clinical features. J Neurosurg 2010;112:640-47

25. Cogliano MA, Tolerico PH. Nonhealing wound resulting from a foreign body to a radial arterial sheath and sterile inflammation associated with transradial catheterization and hydrophilic sheaths. Catheter Cardiovasc Interv 2004;63:104-05

26. Ziakas A, Karkavelas G, Mochlas S. Sterile inflammation after transradial catheterization using a hydrophilic sheath: a case report. Int J Cardiol 2005;99:495-96

27. Kozak M, Adams DR, Ioffreda MD, et al. Sterile inflammation associated with transradial catheterization and hydrophilic sheaths. Catheter Cardiovasc Interv 2003;59:207-13 